\title{
Study on Improved Chaotic Particle Swarm Optimization Based Logistics and Transport Scheduling Problems with Soft Time Windows
}

\author{
Yuqiang Chen ${ }^{1}$, Jianlan Guo ${ }^{2 *}$ \\ Department of Computer Engineering Dong Guan Polytechnic \\ Dongguan, Guangdong, China \\ 1,chenyuqiang@126.com,2,rachel0519@126.com
}

\begin{abstract}
Research the Incident Vehicle Routing Problem with Soft Time-windows (IVRP-STW). Associated logistics scheduling problem with soft time windows using the logistic function optimization method to improve the chaotic particle algorithm, compared with the GA and the standard PSO algorithm. Simulation results show that such optimization method can effectively improve the global search of the particles in the particle swarm and the ability of local search is effective in resolving such problems.
\end{abstract}

Keywords: Chaotic particle swarm optimization algorithm, Soft time windows, Scheduling problem

\section{Introduction}

Modern logistics is the product of economic globalization, but also important services to promote economic globalization. In recent years, China's logistics industry is also developing rapidly. However, there is a big problem in the overall efficiency of China's logistics industry. For example, in 2009, China's logistics expenses accounted for $18 \%$ of GDP, while the U.S. logistics costs amounted to only $9 \%$. If logistics costs to GDP ratio can evaluate the logistics, then we can see that the efficiency of China's logistics industry needs to be improved.

Transportation scheduling problems must be studied to improve the logistics efficiency problem. Transportation with time windows scheduling problem is an important research direction in transportation scheduling problem. Domestic and foreign scholars have conducted a lot of research. G. B. Alvarenga etc. [1] uses partition function and genetic algorithms to study VRPTW, proven heuristic algorithm is better than algorithm. Rita Macedo etc. [2] solve VRP through the establishment of pseudo-polynomial model for multi-path with time windows. Yannis Marinakis etc[3] studied hybrid genetic particle swarm optimization algorithm based VRP. Domestic Chen, Cai Yanguang Li Yongsheng studied the union transport scheduling problem. Qu Yuan, Li Min studied the logistics transport problem with multi-distribution center logistics. They give feasible solutions and have achieved valuable results. In this paper, a soft time windows associated with logistics and transport scheduling problem is as study target to research to improve the chaotic PSO in its application.

\section{Logistics Associated with Soft Time Windows Scheduling Problem Description and Mathematical Model}

Logistics associated with time window divided into two categories: namely, hard time window and soft time windows. The requirement of hard time window is that delivery vehicles must complete delivery under the constraints of customer-specified time window, otherwise delivery fails. The difference between soft time window and hard time 
window is that delivery vehicles are allowed to arrive earlier or postpone to delivery customers. Customer specified time window constraints are relaxed while adding a punishment mechanism to replace the distribution fails. Such a situation logistics and transport process often appears more practical in real life.

\subsection{The Description of Logistics and Transport Scheduling Problems with Soft Time Windows}

Logistics and transport scheduling problems with soft time windows can be described as bellow: a logistics company, a logistics center station, 1 costumers, customer demand for goods $q_{i}(\mathrm{i}=1,2, \ldots, 1), \mathrm{m}$ distribution vehicles, each vehicle's maximum load is $Q_{k}(\mathrm{k}=1,2, \ldots, \mathrm{m})$, delivery vehicles must complete delivery in the time range $\left[A_{i}, B_{i}\right] . d_{i j}$ represents the time cost from costumer $\mathrm{i}$ to costumer $\mathrm{j}$ of a certain delivery vehicle. $S_{i}$ represents the time point of delivery vehicle reaching customer i. $T_{i}$ represents the waiting time cost serving process to costumer $i$. The requirement of soft time windows is that if the vehicle cannot reach the customer in the time window the customer specified, it will have to be subject to certain penalties. If the vehicle reach the customer ahead the time $A_{i}$, the vehicle needs to wait. In the above situation, it will generate time waiting punishment. If the vehicle reach the costumer after the time $A_{i}$, the distribution service has been delayed, it will generate late punishment. Different customers have correlations in distribution time and have correlation constraints. The vehicle starts from the distribution center, distribute the goods to customer and return to the distribution center. The problem is to reasonably arrange the vehicle and distribution routes to lowest the cost under the condition of meeting all the needs of customers.

Constraints are listed as bellow:

(1) Logistics center position is fixed and the only ;

(2) Distribution process is closed, as starting from a logistics distribution center, completing the distribution task and return back;

(3) Delivery vehicles for non-load state, has the largest distribution of load and the maximum distance constraint;

(4) Each customer can only be served by a distribution vehicle and ensure that complete the distribution to each customer.

(5) Vehicle maximum load is known, the customer demand for goods known;

(6) If the delivery vehicle cannot arrive at the customer in the time window, it will pay waiting and late penalties.

(7) Each customer's time window is known.

(8) Customers require simultaneous delivery and pickup ;

(9) Different customers' distribution time have correlation。

\subsection{The Mathematical Model of Associated Scheduling Transportation Problem with Soft Time Window}

Based on the above description of the problem, we can get the mathematical model of associated scheduling transportation problem with soft time window as bellow:

Variables definition :

$$
x_{i j k}=\left\{\begin{array}{lll}
1 & \text { vehicle } k & \text { from } i \text { toj } \\
0 & \text { else }
\end{array}\right.
$$




$$
y_{i k}= \begin{cases}1 & \text { goods of point } \mathrm{i} \text { compl et ed by vehicle } \mathrm{k} \\ 0 & \text { else }\end{cases}
$$

Suppose the distance of $\mathrm{i}$ and $\mathrm{j}$ is $d_{i j}$, the number of distribution center is 0 . The objective is to minimize the total cost of the vehicle distribution. As the major factor of the distribution costs is the distribution distance, the shorter the distance in the distribution process, the lower the time, fuel, labor cost. So this paper selects the delivery distance as the objective function. Customer $i \quad(i=1,2, \ldots, l)$. With soft time window, if the delivery vehicle early arrives at the customer's location, it will get the waiting punishable coefficient of P1. The penalty cost equals $P_{1}\left(A_{i}-S_{i}\right)$. If the delivery vehicle postpones arriving at the customer's location, it will get late penalty $\mathrm{P} 2$. The penalty cost equals $P_{2}\left(S_{i}-B_{i}\right)$.

Objective functions :

$$
\begin{aligned}
& \min z=\sum_{i=0}^{l} \sum_{j=0}^{l} \sum_{k=1}^{m} d_{i j} x_{i j k}+P_{1} \sum_{i=1}^{l} \max \left\{A_{i}-S_{i}, 0\right\}+P_{2} \sum_{i=1}^{l} \max \left\{S_{i}-B_{i}, 0\right\} \\
& T_{i}=\max \left\{A_{i}-S_{i}, 0\right\} \quad i, \quad j \in l \\
& S_{i} \leq B_{i} \quad i, j \in l \\
& \sum_{i=0}^{k} g_{i} y_{i j k} \leq q \\
& i, j \in l \\
& \sum_{n=1}^{N} d_{i j k} \leq D_{\max } \quad i, j \in l \\
& \sum_{i=1}^{l} y_{j k}=1 \quad j \in l, k \in m \\
& \sum_{i=0}^{k} x_{i j k}=y_{i k} \quad i, j \in l, k \in m \\
& \sum_{j=0}^{k} x_{i j k}=y_{i k} \quad i, j \in l, k \in m \\
& \sum_{i=0}^{l} \sum_{j=o}^{l} x_{i j k} \leq l \quad i, j \in l, k \in m \\
& S_{i}<S_{j}, T_{s} \in\{(i, j), i, j \in l\}
\end{aligned}
$$

In the above mathematical model, the formula (1) is the objective function; the function is basing the basic cost formula and adding waiting and late penalty factors. Eq.2 represents the current customer waiting time constraints. The waiting time is determined by the arriving time and the waiting time of the vehicle. Due to the constraint of the soft time window, if the time point of the vehicle reaching the current customer is earlier or late of the time window, then the waiting time of the vehicle can be computed by the time window point minus the reaching time point of the vehicle. It will get waiting penalty, the penalty formula is like ${ }^{P_{1} \sum_{i=1}^{l} \max \left\{A_{i}-S_{i}, 0\right\}}$.If the arriving time is late of the time window, it will get late penalty, and the penalty formula is ${ }_{2} \sum_{i=1}^{l} \max \left\{S_{i}-B_{i}, 0\right\}$. This formula ensure that the logistics company will get penalty if the distribution vehicle arriving at the customer 
earlier or late of the time window [5]. Formula (4) represents that the load of each vehicle is less than the maximum load. Formula (5) is the constraint of the vehicle distance, $d_{i j k}$ is the distance from $i$ to $j$ of vehicle $k, n$ is the number of customers served by vehicle $k$, the maximum number is $N$. Formula (6) ensures each customer can only be served by one vehicle. Formula (7) and (8) represent vehicle $\mathrm{K}$ goes to serve $j$ after serving customer $i$. Ensue the vehicle $\mathrm{K}$ can arrive all the customers at the specific distribution route and each customer is served only one time. Formula (9) represents that customers of each car is less or equal than the total customers. Formula (10) represents that customer $\mathrm{i}$ is served earlier than customer $\mathrm{j}$.

\section{Chaos Particle Swarm Optimization Algorithm of Logistics Scheduling Problem with Soft Time Window}

\subsection{Chaos PMO Algorithm Optimized by Logistic Function}

Inertia factor $\omega$ is an important factor to Chaos PSO algorithm. It affects the convergence and optimization of the Chaos PSO algorithm process. When each particle in Chao PSO approaches the target value or close to the local optimal solution, the inertia factor will increase, otherwise the inertia will decrease. Particles whose target value is better than the average target value will correspond the smaller inertia factor. Such particles will be saved. Particles whose target value is much different with the average target value will correspond the bigger inertia factor. Such particles will fastly trend to the better searching space. Basing the above idea, this paper proposes a method which using Logistics function to adjust inertia factor [4].

Logistics function is also called S-function or compression function, its function is like (11).

$$
y=a+\frac{b}{1+e^{-d x}}
$$

From Eq. 11, we can see that Logistics function is nonlinear and differentiable. It has good gain control to the inputting variable. The constants in the function can be defined according to different circumstances, so that get different gain controlling effect under different circumstances. When the input value is small, the output is big, otherwise the output is small. All the above characteristics meet the requirements to make nonlinear adjustment to inertia factor.

Basing the above characteristics of Logistics function, this paper uses Eq. 12 to make nonlinear adjustment to the inertia function ${ }^{\omega}$.

$$
\omega_{i}=\omega_{\max }-\left(\omega_{\max }-\omega_{\min }\right)\left(\frac{2}{1+e^{-d_{i} \frac{N_{i}}{N_{\max }}}}-1\right)
$$

$\omega_{\max }, \omega_{\min }$ are the max and min value of $\omega, N_{\max }$ is the maximum interaction number, $N_{i}$ is the current iteration number. $\omega_{i}$ is the inertia of the ith inertia factor. The value of $d_{i}$ in the above function is determined by function (13).

$$
d_{i}=c \xi_{i}, \xi_{i} \in(0,1), i \in[0, n]
$$

Constant $\mathrm{c}$ is determined according to the circumstance which is the chaos variable of the ith iteration. $\mathrm{N}$ is the number of the particle swarm populations.

Use Logistic function to make nonlinear adjustment to the inertia weight factor to decrease the overall changing speed of the inertia factor. Due to each swarm is optimized by different inertia factor, each swarm has unique trajectory in the iteration process. The advantage is that the swarms have optimal overall and local searching ability and improve the optimization ability. 


\subsection{Algorithm Process}

First, use the standard PSO to generate swarms to the preliminary solution. Then, use chaos mapping to make chaos optimization and use Logistic function to make nonlinear adjustment to the inertia factor to get the optimization solution. The algorithm process is like following.

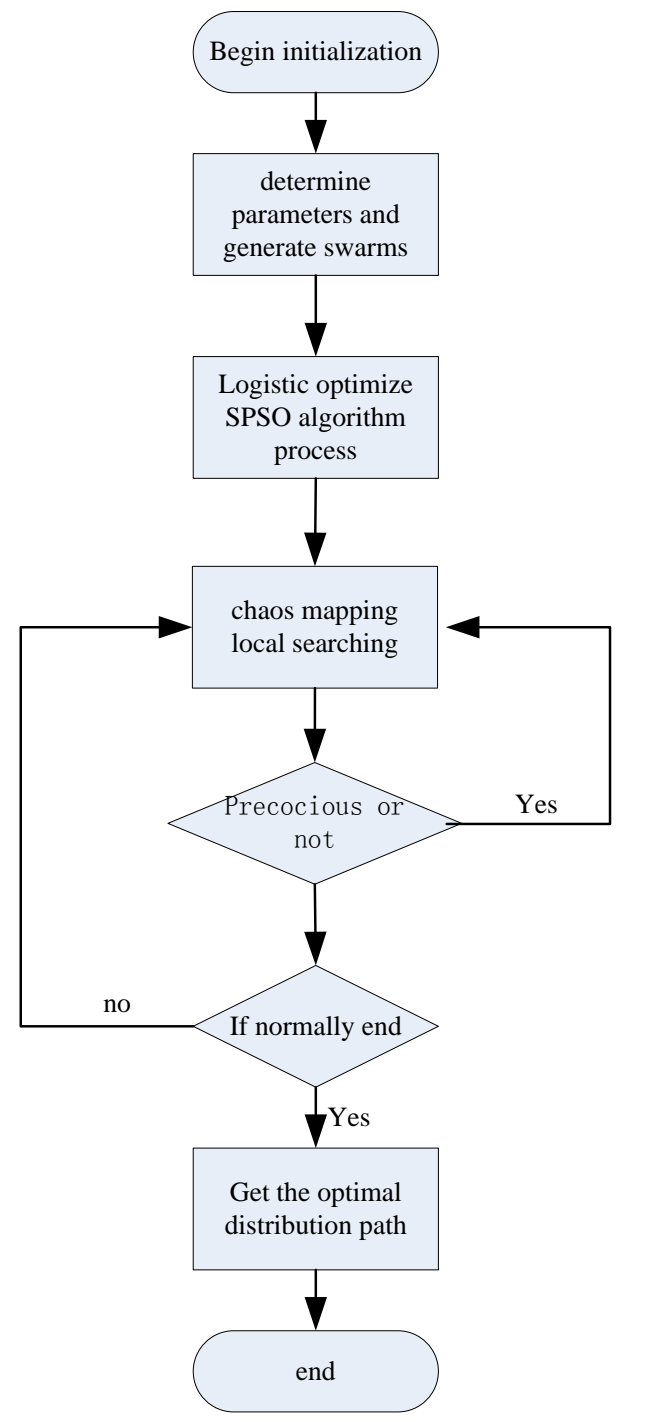

\section{Figure 1. CPSO Algorithm Flowchart}

The algorithm process is like following:

Step 1 Initialization. Initialize the particle swarm; input the basic data of the distribution network. Determine the swarm scale n, learn factor $c_{1} c_{2}$, the max and min value of $\omega, \omega_{\max }, \omega_{\min }$, penalty coefficient $\mathrm{p}$, the max iteration time $N_{\max }$

Step 2 Generate swarm. According the basic data of the distribution network to generate the particle swarm with scale $\mathrm{n}$.

Step 3 Compute the inertia weight factor using Eq.11 and Eq.12.

Step 4 Execute the standard PSO algorithm, reserves the particles with preliminary good performance. Get $p_{g}=\left(\begin{array}{lll}p_{g 1} & , p_{g 2}, \ldots p_{g m}\end{array}\right)$ which is the same as $p_{\text {best }}$. 
Step 5 Make chaos optimization to the above reserved particles, get chaos sequence. Then make chaos mapping, complete the chaos optimization procedures. Get $p_{g, k}^{* 1}$ and update the position of optimal solution.

Step 6 Precocious judgment. Determine whether the particle swarms fall in premature convergence, if premature then go back to procedure 4, if no premature then continue the algorithm. There are two obvious features of premature particles. The first is serious gathered and the other is no changing after number of iterations. Using Eq.14 to compute the group fitness variance.

$$
\omega=1.5-\frac{1}{1+r_{1} \exp \left(-r_{2} \cdot \square\right)}
$$

Compare with the predefined minimum group fitness variance $\sigma_{\min }^{2}$, if $\sigma^{2} \leq \sigma_{\min }^{2}$, then the swarm is serious gathered.

Step 7 The stop condition of the judgment algorithm. Compute the optimal iteration number of $p_{g . k}^{* l}$. If the iteration number is bigger than the maximum iteration number, then the chaos optimization stops.

Step 8 Get the global optimal solution which is the optimal distribution path.

Step 9 Algorithm end.

\section{Experiment Results and Analyze}

The configuration of the PC used in our experiment is Intel(R) Core $2 \mathrm{CPU} 2.66 \mathrm{GHz}$ Memory 2.0G. The software is Matlab 7.0. The experiment is like following.

A logistic center has a distribution center which is 0 . The number of customers is 9 . The relationship between distribution center, good demand of each customer and soft time window is shown in table 4-1. The speed of distribution vehicle is 50 which maximum load 10 ton. Unit good distribution cost is 1 . The distribution service time is 5 . Waiting penalty factor $P_{1}=2$. Late penalty factor $P_{2}=3$. It is required that customer 5 is served earlier than customer 6 . The final goal is to compute the optimal distribution path.

Table 1. Customer Demand, Service Time and Time Window Data

\begin{tabular}{c|c|c|c|c|c}
\hline Customer & 0 & 1 & 2 & 3 & 4 \\
\hline Demand & 3 & 2 & 1.5 & 1.9 & 5 \\
\hline Position & $(99,39)$ & $(39,70)$ & $(22,15)$ & $(97,79)$ & $(6,28)$ \\
\hline $\begin{array}{c}\text { Time } \\
\text { Window }\end{array}$ & {$[0,0]$} & {$[7,12]$} & {$[5,10]$} & {$[1,6]$} & {$[9,14]$} \\
\hline Customer & 5 & 6 & 7 & 8 & 9 \\
\hline Demand & 4.2 & 3.3 & 0.9 & 1.4 & 5.3 \\
\hline Position & $(62,79)$ & $(42,88)$ & $(86,3)$ & $(87,61)$ & $(88,90)$ \\
\hline Time & {$[4,9]$} & {$[8,13]$} & {$[2,8]$} & {$[1,5]$} & {$[3,9]$} \\
Window & & & & & \\
\hline
\end{tabular}

The basic parameters used in our experiment are like: Number of particle population $50, \mathrm{c} 1=1, \mathrm{c} 2=1$, adaptive inertia factor $w_{\text {max }}=0.9, w_{\text {min }}=0.1$, the maximum iteration time is 150 .

The optimal distribution paths generated by our experiment are like following.

Distribution path 1:0-8-3-9-0,

Distribution path 2:0-7-2-4-0,

Distribution path 3:0-5-6-1-0.

Optimal total distribution $\cos t=512.5$. 
The total distribution cost includes two parts, basic logistic cost 493.6 and penalty cost 20.8 which occurred in the distribution for customer 6 .

To validate the performance of our algorithm, we use standard GA and PSO to run the experiment 30 times. The population number is 50 . The iteration is 150 . The experiment result is like Figure 2.

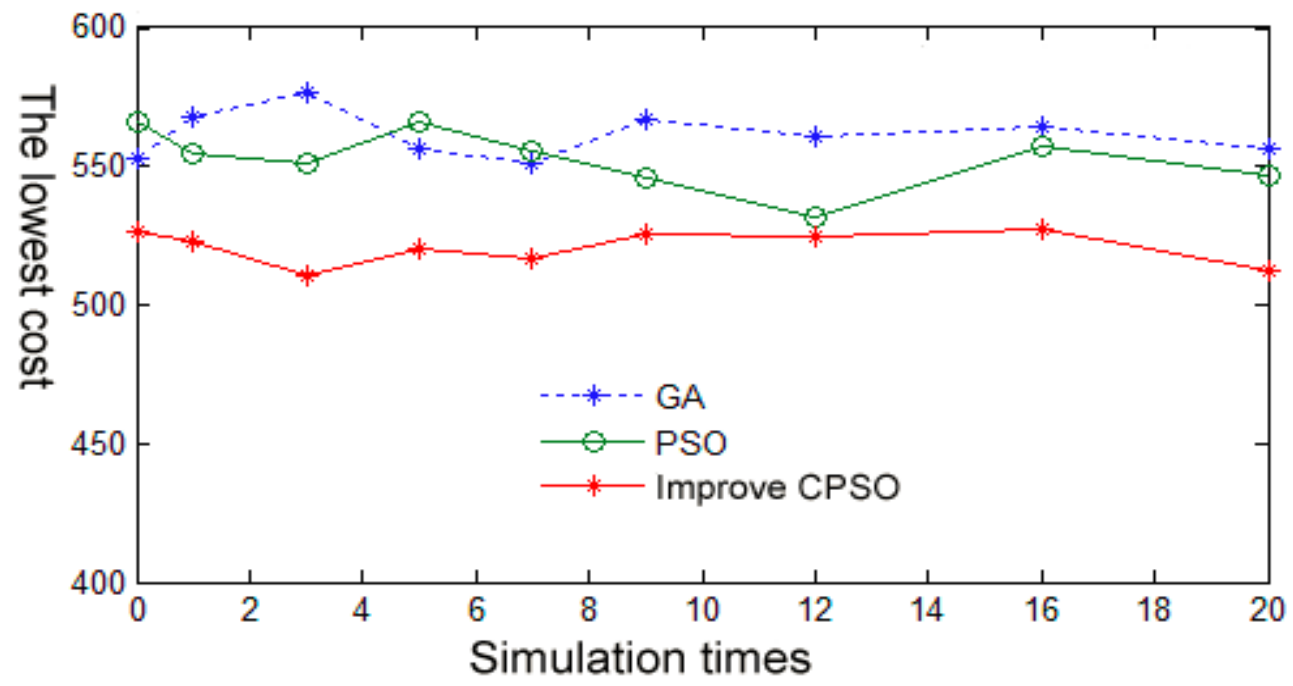

Figure 2. Comparison of the Three Algorithms

From the above experiment results we can conclude that the objective function will be complex with adding soft time window for logistics scheduling problem. The improved chaos PSO is more stable than GA and PSO algorithms

\section{Summary}

This paper introduces the logistics scheduling problem with soft time window. Construct mathematical model. Introduce Logistic function optimization chaos PSO algorithm. The algorithm let the particles have better global and local searching ability and expand the scope of the solution space. The do experiment in Matlab platform. Experiment results show that our proposed algorithm and effetely solve the problem.

\section{Acknowledgements}

The work described in this paper was supported by National Natural Science Foundation of China (No.61106019), also supported by Guangdong Provincial of Science and Technology Foundation (No. 2012B040500007), also supported by the excellent young teachers program project of Guangdong Province(No:Yq2013201), and also supported by the project of Chinese Institute of logistics (2014CSLKT3-205).

\section{Reference}

[1] Y. Marinakis and M. Marinaki, "Expert System with Applications", vol. 37, (2010).

[2] R. Macedo, C. Alves, J. M. V. Carvaiho, F. Clautiaux and S. Hanafi, "European Journal of Operational Research", vol. 214, (2011), pp. 536-545.

[3] G. B. Alvarenga, G. R. Mateus, G. D. Tomi, "Computers \& Operations Research", vol. 34, (2007), pp. 1561-1584.

[4] S. Suresh, P. B. Sujit and A. K. Rao, "Composite Structures", vol. 81, no. 4, (2007), pp. 598-605.

[5] A. E1-Gohary and A. S. A1-Ruzaiza, "Chaos, Solitons and Fractals", vol. 34, no. 2, (2007), pp. 443-453.

[6] X. H. Shi, Y. C. Liang, H. P. Lee, et. al., "Information Processing Letters vol. 103, no. 5, (2007), pp. 169-176. 
[7] Z. Yi, H. L. Hai long and W. Rui, "Journal of Jilin University", Science Edition vol. 02, (2010), pp. 197-203.

[8] J. Chen and Y. G. Cai, "Industrial Control Computer", vol. 9, no. 3, (2009), pp.70-72.

\section{Authors}

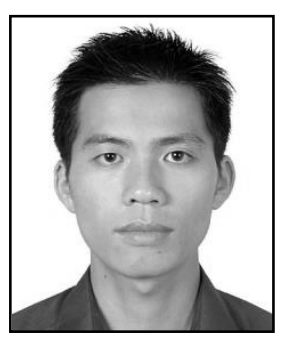

Chen Yuqiang, was born in 1980, graduated from Guangdong University of Technology, and is associate professor in Dongguan polytechnic College. His present research interests include automatic control and computer applications.



Guo Jianlan, was born in 1984, graduated from Guangdong University of Technology, and is senior engineer in Dongguan polytechnic College. Her present research interests include automatic control and computer applications. 